\title{
La historia del arte como ciencia: La inserción de Imre Lakatos en la definición de la disciplina
}

\section{(The history of art as a science: Imre Lakatos insertion in the definition of the discipline)}

\author{
Mauricio Oviedo SALAZAR
}

Recibido: 28 de enero de 2015

Aceptado: 7 de octubre de 2015

\section{Resumen}

El artículo pretende definir la Historia del Arte dentro del campo de la ciencia. Entenderemos ciencia bajo el amparo de las teorías del filósofo de la ciencia Imre Lakatos. El texto quiere demostrar la posibilidad de pensar la Historia del Arte desde el punto de vista racional, con la idea de que es posible fabricar tanto reconstrucciones racionales históricas relativas a lo que la disciplina estudia, como una reconstrucción racional de la historiografía del arte.

Palabras clave: Historia del Arte, Epistemología, Metodología, Ciencia Social.

\begin{abstract}
This article tries to define Art History in the field of science. We will understand science under the aegis of the theories of the philosopher of science Imre Lakatos. The text tries to show the possibility of thinking Art History from a rational point of view, under the assumption that it is possible to provide a rational historical reconstructionof the discipline's object of study, as well as a rational reconstruction of the historiography of art.
\end{abstract}

Keywords: Art History, Epistemology, Methodology, Social Science. 
Las humanidades, en las que se inscribe la historia del arte, disciplina a la cual le dedicaremos este artículo, han caído en un juego de mal gusto. Por ser de carácter social sus estudios relativos al conocimiento humano y a su entorno, nos es supuestamente permitido utilizar de forma indiscriminada cualquier programa de investigación o metodología. Sin la presencia de un análisis crítico y reflexivo tocante a dicho programa, no nos es posible exponer de qué forma ha progresado o ha degenerado. Esta actitud, por ejemplo, anula la función del estado de la cuestión en los estudios de carácter histórico, y por lo tanto nos hace más compleja la separación entre los enunciados básicos relativos a un tema que entran en conflicto con nuestra propuesta, y los que no. Las metodologías de las ciencias sociales y de las humanidades han pasado a ser simples herramientas de interpretación, que abusan de las palabras 'tolerancia' y 'respeto', entre otras, para así poder escapar de la contienda por la generación de conocimiento. Como resultado de tan deplorable situación, se han convertido en disciplinas de opinión expertas en el uso de argumentos confusos, relativistas, absurdos, y en muchos casos irracionales. 12

Ante este escenario presentamos la siguiente discusión, la cual es un primer intento de definir la historia del arte, en cuanto que disciplina académica, como ciencia, es decir, bajo la consideración de que es una herramienta para el aumento del conocimiento. Se busca plantear la posibilidad de realizar una reconstrucción racional de la historiografía del arte: cómo, mediante la percepción de la disciplina como ciencia, podemos comprender sus diversos procesos históricos a partir de la consideración de cambios progresivos y cambios regresivos de problema. Dichas aseveraciones se realizan teniendo como base al filósofo de la ciencia húngaro Imre Lakatos (1922-1974), y en un menor grado, de ciertos elementos propuestos por el filósofo de la ciencia austro-británico Karl Popper (1902-1994). Empero, esto no significa que tanto Lakatos como Popper hayan tratado problemas relativos a la his-

\footnotetext{
1 A esto podemos añadir la confusión que hay entre hacer historia y practicar el historicismo. En conformidad con Popper (2006, p. 17) entenderemos historicismo como: “... un punto de vista sobre las ciencias sociales que supone que la predicción histórica es el fin principal de éstas, y que supone que este fin es alcanzable por medio del descubrimiento de los 'ritmos' o los 'modelos', de las 'leyes' o las 'tendencias' que yacen bajo la evolución de la historia” (Popper, 2006, p. 17).

Para el filósofo, el historicismo es en gran medida culpable de que: “... en las ciencias sociales no haya casi nada parecido a la objetividad y al ideal de búsqueda de la verdad que vemos en la física. Es de esperar que nos encontremos en las ciencias sociales con tantas opiniones como se puedan encontrar en la vida social, tantos puntos de vista como hay intereses. Se puede preguntar si este argumento historicista no nos lleva a esa extrema forma de relativismo que sostiene que la objetividad y el ideal de veracidad son totalmente inaplicables a las ciencias sociales donde sólo el éxito -el éxito políticopuede ser decisivo" (Popper, 2006, p. 30).

2 Podemos considerar un argumento irracional como aquel que no posea la propiedad de falsabilidad, es decir, uno en el que no se ofrece la posibilidad de diseñar un protocolo en el que este pueda contrastarse ante falsadores potenciales. Al contrario, los argumentos racionales deben dar la posibilidad de poder ser contrastados con el mundo empírico (Oviedo, 2014, p. 20).
} 
toria del arte, en realidad dedicaron su carrera intelectual a la historia de la ciencia y a los problemas de la epistemología, concebida como disciplina filosófica de carácter normativo, atinente al incremento del conocimiento. 34

Asimismo, debemos comprender que si bien las propuestas de Popper son acogidas para la presente definición, estas se someten a lo expuesto por Lakatos. ${ }^{5}$ Esto implica que en determinados momentos nos será necesario dejar claro si alguna de las posiciones, tesis o aseveraciones del filósofo austro-británico no son utilizadas o seguidas por nuestro programa, con el fin de no caer en inconsistencias de carácter metodológico. Ante todo, la definición de historia del arte se va a supeditar a los programas de investigación, los conceptos popperianos incluidos son los retomados por Lakatos.

\section{La historia del arte como ciencia: una primera definición}

En términos generales, nuestra definición se expone de esta manera: la historia del arte es aquella ciencia que estudia los objetos artísticos ${ }^{6}$ como problemas histó-

\footnotetext{
3 El problema de la epistemología se ha abordado en muchos casos como un problema del conocimiento del sentido común, y en otros como del conocimiento científico. En el primero se ve el conocimiento científico como una extensión del conocimiento del sentido común. El correspondiente al sentido común se formula por medio del lenguaje ordinario, y por lo tanto dicho lenguaje debe ser analizado. Popper (2004, p. 19) estuvo de acuerdo con la idea de que el conocimiento científico era una aplicación del relativo al sentido común, pero consideró que limitarse al último tenía como resultado la omisión de los problemas más importantes para la epistemología. ¿Cuál sería uno de esos problemas tan significativos a los que Popper aludía? El del aumento del conocimiento: "Basta una ligera reflexión para convencerse de que la mayoría de los problemas que se encuentran en conexión con dicho aumento han de trascender, necesariamente, todo estudio que permanezca confinado en el conocimiento de sentido común, frente al conocimiento científico: pues la manera más importante de aumentar aquél es, precisamente, volviéndose conocimiento científico. Y, además, parece evidente que el aumento de este último es el caso más importante y más interesante del aumento de los conocimientos" (Popper, 2004, p. 19).

4 Es claro que si estos dos pilares de la filosofía de la ciencia se convierten en nuestra base para el desarrollo de nuestra propuesta, estamos entonces tomando de ellos las aproximaciones a la ciencia desde lo que conocemos como: metodología para los programas de investigación científica, falsacionismo metodológico sofisticado y método hipotético deductivo. Debe quedar claro que ninguno de estos tres términos denota lo mismo, son tres expresiones que establecen una serie de conexiones entre sí, y que a su vez cada una de las tres nociones descarta determinadas implicaciones que se siguen de las otras dos. Por esta razón es necesario decir que la definición de historia del arte como ciencia que desplegaremos, se supedita, con sus reservas las cuales serán indicadas en el texto, a las ideas de programas regresivos y progresivos de investigación, falsación y deducción.

5 En realidad nos estamos sirviendo de un Popper lakatosiano.

${ }^{6}$ En el presente artículo no nos detendremos a definir objeto artístico, ya que nos desviaríamos del objetivo por el cual realizamos este texto. No obstante, en términos generales, nos encontramos en gran medida de acuerdo con lo propuesto por el historiador del arte alemán Erwin Panofsky, en su artí-
} 
ricos. ${ }^{7}$ Tal oración nos obliga a precisar 'historia', la cual sería, de forma en sumo concisa, la ciencia que examina, por vía principalmente de fuentes textuales, ${ }^{8}$ el pasado referente al ser humano y a su entorno, ${ }^{9}$ a partir principalmente de acontecimientos concretos y de personalidades individuales, los cuales conforman los aspectos elegidos del objeto de investigación por el cual está interesado el investigador (Popper, 2006, p. 96):10 "Toda historia escrita es la historia de un cierto

culo "On the Relationship of Art History and Art Theory: Towards the Possibility of a Fundamental System of Concepts for a Science of Art", publicado en 1925, en el que el autor escribió que la obra de arte, o el objeto artístico, es un elemento que proporciona soluciones para problemas artísticos en particular. Estos problemas se muestran como opuestos, y es en el "objeto artístico" donde se concilian. Tales problemas son subsumibles en problemas mayores, adscritos al volumen y a la forma, principios a priori, a su vez polos opuestos, quienes por lo tanto son unidos, de alguna forma en particular, por el mismo "objeto". Asimismo, tanto el volumen como la forma, en cuanto a principios a prio$r i$, están relacionados al espacio y el tiempo, por lo que podemos decir que el volumen corresponde al espacio, y la forma al tiempo. El volumen y la forma son necesarios, a priori, para que existan problemas artísticos; en este sentido el espacio y el tiempo juegan el papel de condición a priori para la solución de dichos problemas, es decir para la creación del "objeto artístico". Entonces, el volumen y la forma son problemas que están en un constante roce, el espacio y el tiempo se unen para ofrecer diversas soluciones a los disímiles problemas artísticos causados por la constante interacción entre volumen y forma, soluciones plasmadas en el objeto que entendemos como obra de arte (Panofsky, 2008, 4647). Gran parte de estas propuestas son desarrolladas de forma más amplia en el artículo del historiador del arte Edgar Wind, titulado "On the Systematics of Artistic Problems", publicado también en 1925, texto que formó parte de su tesis doctoral. De hecho, en su texto, Panofsky (2008, p. 45) se muestra en deuda con lo que Wind estaba desarrollando.

${ }^{7}$ En conformidad con Agassi y Jarvie (2008, pp. 133-134), es mejor estudiar el arte en contexto, entendiendo contexto como el mundo de arte y su propia integración en la sociedad. Para sustentar dicha afirmación se dirigen al historiador del arte Ernst Gombrich, quién corrige la típica pregunta que se formula respecto de la obra de arte: ¿La comprensión de la obra de arte requiere tener conocimiento de las intenciones del artista? Gombrich propone una pregunta más acertada: ¿Cuál fue el contexto en el que la obra de arte fue creada? Agassi y Jarvie continúan esta idea al establecer que disímiles artes y disímiles audiencias, nos llegan a proporcionar diferentes tipos de experiencia, elementos que debemos de tomar en cuenta a la hora de acercarnos a un objeto artístico como problema histórico.

8 Tanto fuentes textuales primarias cuanto fuentes textuales secundarias.

9 Para Popper (2006, pp. 47-48), la Historia es la descripción del cambio, y es solo a través de ella que podemos comprender el carácter de un grupo social: “... si los grupos sociales sólo pueden ser conocidos a través de su historia, los conceptos que se usen para describirlos tienen que ser conceptos históricos; y de hecho, conceptos sociológicos como el Estado japonés o la Nación italiana o la Raza aria no puede ser interpretados más que como conceptos basados en el estudio de la historia. Lo mismo vale para las clases sociales: la burguesía, por ejemplo, sólo puede ser definida por su historia: como la clase que llegó al poder por medio de la revolución industrial, que echó a un lado a los propietarios de tierras y que está combatiendo y siendo combatida por el proletariado, etc." (Popper, 2006, pp. 4748).

10 De acuerdo con Popper (2006, p. 96): "Es una equivocación el creer que puede haber una historia en el sentido holístico, una historia de 'Estados de la Sociedad' que represente 'la totalidad del organismo social' o 'todos los acontecimientos históricos y sociales de una época'. Esta idea deriva de una visión intuitiva de una historia de la humanidad como una vasta y comprensiva corriente de desarro1lo. Pero una historia de esa clase no puede ser escrita" (Popper, 2006, p. 96). El filósofo posterior- 
aspecto estrecho a (...) [el] desarrollo 'total', y es de todas formas una historia muy incompleta incluso de ese particular aspecto incompleto que se ha escogido." (Popper, 2006, p. 96).

También resulta necesario decir que lo que entendemos por ciencia (scientia) es el conocimiento, pero este no se traduce en la convicción que un gran número de individuos tenga sobre la "verdad" de una teoría. Creer en una hipótesis no es el equivalente a conocer. Lo que debe imperar en el científico (aquel dispuesto a conocer), es un cierto grado de escepticismo hasta con las teorías más fuertes. Hemos de diferenciar, de forma clara, al conocimiento de la superstición, ideología o pseudo ciencia (Lakatos, 1983, pp. 9-10), por razón de que la creencia o convicción en algo no es un criterio de demarcación satisfactorio:

(...) un enunciado puede ser pseudocientífico aunque sea eminentemente plausible y aunque todo el mundo lo crea, y puede ser científicamente valioso aunque sea increíble y nadie crea en él (...)

El valor cognoscitivo de una teoría nada tiene que ver con su influencia psicológica sobre las mentes humanas. Creencias, convicciones, comprensiones... son estados de la mente humana. Pero el valor científico y objetivo de una teoría es independiente de la mente humana que la crea o la comprende. ${ }^{11}$ Su valor científico depende solamente del apoyo objetivo que prestan los hechos a esa conjetura. (Lakatos, 1983, p. 10)

Imre Lakatos (1983, p. 12) escribió que una teoría tiene la capacidad de ser científica aun si no cuenta con evidencia a su favor, y puede obtener el calificativo de pseudo científica aunque tenga toda la evidencia disponible de su lado. Esto significa que no necesitamos, en principio, de los hechos para poder determinar la cientificidad o pseudo cientificidad de una teoría. Debemos, de acuerdo con lo que propuso Popper según Lakatos, examinar si la teoría detalla por adelantado un experimento u observación que esté en capacidad de falsarla. Si este es el caso, estamos más próximos a una teoría cuyo método podría categorizarse como científico, lo que nos permitiría decir que una teoría en la que el método es pseudo científico, o, en todo caso, no científico, es aquella que no especifica dicho falsador potencial.

mente reitera que “(...) la historia se caracteriza por su interés en acontecimientos ocurridos, singulares o específicos, más que en leyes o generalizaciones" (Popper, 2006, p. 161).

${ }^{11}$ Con respecto de esta cita de Lakatos, podemos incluir la percepción que tuvo Popper (2006, pp. 157158) en relación con la conflictiva necesidad que hay para ciertos historiadores y científicos de que, en orden a realizar una descripción de una situación social, debamos considerar la del estado mental y físico de todos los participantes de dicha situación: "Esta creencia también muestra la huella de la opinión popular de que las entidades sociales, como, por ejemplo, las instituciones o asociaciones, son entidades naturales concretas de la misma manera que una aglomeración de hombres, más que modelos abstractos construidos para interpretar ciertas relaciones, abstractas y seleccionadas, entre individuos" (Popper, 2006, pp. 157-158). 
En este sentido, asimismo, hemos sido testigos de que al hablar de ciencia se genera comúnmente una interpretación errónea que peca de ignorancia, la cual propicia la problemática concepción de que los que se amparan bajo esta palabra son poseedores de la verdad absoluta y probada. Pero en realidad, aquellos que se creen los propietarios de lo veraz no son más que personajes que han caído en un pensamiento dogmático, ciego, y por lo tanto, estancado en cuanto al objetivo de adquirir conocimiento. Esta noción de ciencia como 'verdad probada' iría en contra del método hipotético deductivo que hemos adoptado, ya que en él no es posible obtener la evidencia definitiva para las proposiciones científicas que se presentan, o sea, nunca se pierde el carácter hipotético de las propuestas (Popper, 2006, pp. 148-149). En conformidad con Popper (2004, p. 262):

La ciencia nunca persigue la ilusoria meta de que sus respuestas sean definitivas, ni siquiera probables; antes bien, su avance se encamina hacia una finalidad $-y$, sin embargo, alcanzable-: la de descubrir incesantemente problemas nuevos, más profundos y más generales, y de sujetar nuestras respuestas (siempre provisionales) a contrastaciones constantemente renovadas y cada vez más rigurosas.

De acuerdo con este rasgo de la ciencia, tenemos que olvidarnos de las hipótesis o teorías probadas, ya que estaríamos implicando que son irrefutables, por lo que caeríamos en el dogma comentado con anterioridad. Si seguimos estas líneas de Popper (2006, p. 138), nos comprometemos con el hecho de ser investigadores en búsqueda de la creación y resolución constante de problemas. ${ }^{12}$

\section{La historia del arte como una ciencia empírica}

Con lo mencionado hasta el momento, hemos de ver la historia del arte como una ciencia empírica, ${ }^{13}$ y no como un sistema metafísico, por lo cual necesitamos

\footnotetext{
12 En conformidad con el filósofo austro-británico: "La ciencia, puede decirse, trabaja en todo momento sobre problemas. No puede empezar con observaciones o 'coleccionando datos', como creen algunos estudiosos del método. Antes de que podamos recolectar datos debe despertarse en nosotros un interés por datos de una cierta clase: el problema siempre viene en primer lugar. A su vez el problema puede ser sugerido por necesidades prácticas o por creencias científicas o precientíficas que por una u otra razón parecen necesitar una revisión. Ahora bien, un problema científico, por regla general, nace de la necesidad de una explicación" (Popper, 2006, p. 138).

13 Popper (2004, pp. 27-28) se opuso a la noción de vincular las ciencias empíricas a los "métodos inductivos", relación que hacía de la lógica de la investigación científica un equivalente a la lógica inductiva. Podemos entender como 'inductivo' al momento en que los enunciados singulares, como descripciones de los que resultó de la observación o de los experimentos, pasan a ser enunciados universales, como las hipótesis o los sistemas de teorías. Pero la inducción nos hace preguntarnos bajo qué condiciones están justificadas las inferencias inductivas, ¿es posible hablar de la verdad de los
} 
tener claro cuál es el criterio de demarcación que nos diferencia como tal (Popper, 2004, p. 34). Por ahora tengamos presente para el desarrollo de este texto, que una primera parte del criterio de demarcación que adoptaremos es la exigencia de falsabilidad de los sistemas, ${ }^{14}$ lo que involucra que para que un sistema sea empírico debe de ser posible poder contrastarlo, y por lo tanto, refutarlo a través de la experiencia (Popper, 2004, p. 40), es decir, ha de ser empíricamente falsable, o en otras palabras, su falsabilidad debe ser empírica. Los enunciados deducidos del sistema teórico propuesto han de ser por lo tanto objetivos, entendiendo objetivo como justificable, 15 que esté en capacidad de ser contrastado y comprendido por cualquiera (Popper, 2004, p. 43).

¿Es posible poder contrastar y refutar los sistemas vinculados a la historia del arte? ¿Es viable visualizar los enunciados de un sistema teórico relativo a la histo-

enunciados universales al estar basados estos en la experiencia? ¿Podemos decir que la verdad se 'sabe por experiencia'? Para Popper todo aquello que sea resultado de la experiencia (observación y experimento) no es en principio un enunciado universal, sino solamente un enunciado singular: “... quien dice que sabemos por experiencia la verdad de un enunciado universal suele querer decir que la verdad de dicho enunciado puede reducirse, de cierta forma, a la verdad de otros enunciados -éstos singulares- que son verdaderos según sabemos por experiencia; lo cual equivale a decir que los enunciados universales están basados en inferencias inductivas" (Popper, 2004, p. 28).

Para intentar justificar la inferencia inductiva debemos poder establecer primero un principio de inducción, el cual es un enunciado que permitiría que proporcionemos las inferencias de una manera lógicamente aceptable. Para los defensores de la inducción, el principio de inducción nos da la verdad de las teorías científicas, sin él no podríamos discernir la verdad o falsedad de una teoría científica. Pero este principio no puede ser una verdad en su totalidad lógica, ya que eliminaría el problema de la inducción, y nos llevaría a considerar que toda inferencia realizada por medio de la inducción son por entero lógico-deductivas. El principio de inducción, en su calidad de verdad puramente lógica, tendría que entenderse como un enunciado universal, pero esto nos llevaría a una regresión infinita no inocua ya que, para justificar el enunciado universal hemos de utilizar inferencias inductivas, basados en la idea de que sabemos por experiencia la verdad de los enunciados, pero para justificar las inferencias inductivas tendríamos que tener clara la existencia de un principio de inducción, y esto repetiría, regresiva e infinitamente, el proceso (Popper, 2004, pp. 28-29). En La lógica de la investigación científica, Popper (2004, p. 30) propone la teoría del método deductivo de contrastar, en el que las hipótesis solamente están en capacidad de ser contrastadas de forma empírica.

14 Posteriormente completaremos dicho criterio.

15 Las intenciones o deseos del investigador no tienen nada que ver con justificaciones objetivas, solo su capacidad de ser contrastadas con la experiencia les dará tal calificativo (Popper, 2004, p. 43). En conformidad con Popper (2004, p. 45): "Por intenso que sea un sentimiento de convicción nunca podrá justificar un enunciado. Por tanto, puedo estar absolutamente convencido de la verdad de un enunciado, seguro de la evidencia de mis percepciones, abrumado por la intensidad de mi experiencia: puede parecerme absurda toda duda. Pero, ¿aporta, acaso, todo ello la más leve razón a la ciencia para aceptar mis enunciados? (...) La única respuesta posible es que no, y cualquiera otra sería incompatible con la idea de la objetividad científica (...) desde el punto de vista epistemológico, carece enteramente de importancia que mi sentimiento de convicción haya sido fuerte o débil, que haya procedido de una impresión poderosa o incluso irresistible de certeza indudable (...) o simplemente de una insegura sospecha: nada de todo esto desempeña el menor papel en la cuestión de cómo pueden justificarse los enunciados científicos." 
ria del arte como objetivos, y por lo tanto justificables? Nuestra respuesta es que sí. La disciplina no solo cuenta con fuentes escritas primarias y secundarias para poder contrastar lo que propone una teoría, sino que tenemos como evidencia las mismas obras de arte, con las cuales la teoría deberá confrontarse en función de ser corroborada o, en todo caso, falsada.

Una teoría empírica (falsable) será aquella que esté en condiciones de dividir en dos clases todos los posibles enunciados básicos: la primera clase contiene los enunciados básicos con los que la teoría no es compatible (y que por lo tanto excluye), esta es la clase de los posibles falsadores de la teoría. De ella nos es posible deducir que, para que la teoría sea empírica debe formularse en principio haciendo explícitas las formas en que puede ser falsada. Ninguna teoría se formula dotada ya de antemano de certeza, esto eliminaría su carácter empírico. La segunda clase contiene los enunciados básicos con los que la teoría no entra en contradicción. Para el filósofo austro-británico, la teoría, y por lo tanto el teórico, debe de hacer afirmaciones solamente sobre la clase de posibles falsadores; en el caso de los enunciados que no están en contradicción, o sea que están permitidos, no dice nada, especialmente en ningún momento afirma que sean verdaderos. Ahora bien, debemos rechazar esta última proposición de Popper, ya que, en línea con los programas de investigación científica de Lakatos, hemos de considerar los enunciados básicos que no entran en contradicción, por razón de que estos nos posibilitan comprender ya sea el cambio progresivo o regresivo de problemática que existe en el programa. Finalmente, la teoría resultará falsada solamente si una hipótesis empírica incompatible con la teoría es corroborada empíricamente. Esta hipótesis la conoceremos como hipótesis falsadora (Popper, 2004, pp. 82-83).

Debemos pensar toda proposición de la ciencia como una proposición teórica falible. En esta noción de ciencia cabe perfectamente el falsacionismo metodológico, el cual es convencionalista 16 revolucionario, por causa de que toma en cuenta la tradición de las teorías del conocimiento 'activas'. ${ }^{17}$ En ellas se considera que no es

\footnotetext{
16 En conformidad con Lakatos (1903, p. 138): "El convencionalismo permite la construcción de cualquier sistema de casillas que organice los hechos en algún todo coherente. El convencionalismo decide mantener intacto el centro de tal sistema de casillas mientras ello sea posible: cuando una invasión de anomalías plantea dificultades, cambia y complica las estructuras periféricas. Pero el convencionalista no considera a ningún sistema de casillas como verdadero por haber sido probado, sino sólo como 'verdadero por convención' (o posiblemente como ni verdadero ni falso, incluso)."

17 Lakatos (1983, p. 32) hizo una distinción entre los activistas conservadores y los activistas revolucionarios, Popper cabe dentro del último término: “... los 'activistas' conservadores sostienen que nacemos con nuestras expectativas básicas; mediante ellas convertimos el mundo en 'nuestro mundo' y debemos después vivir para siempre en la prisión de nuestro mundo. La idea de que vivimos y morimos en la prisión de nuestros 'marcos conceptuales' fue desarrollada en primer lugar por Kant; los kantianos pesimistas pensaron que el mundo real siempre será incognoscible debido a nuestra condición de prisioneros, mientras que los kantianos optimistas pensaron que Dios creó nuestro marco conceptual de modo que se ajustara al mundo. Pero los activistas revolucionarios entienden que los mar-
} 
posible aproximarse a la naturaleza sin antes contar con procesos mentales que nos posibiliten interpretarla de acuerdo con nuestras teorías y expectativas, 18 y crear así constantemente 'verdades por convención' o 'verdades temporales' (Lakatos, 1983, p. 27). Los enunciados aceptados por acuerdo son espacio-temporalmente singulares, y no universales, el valor 'verdadero' que se le dé a dichos enunciados se decide una vez más por acuerdo (Lakatos, 1983, p. 34). La base empírica es verdadera no por esencia sino, antes bien, por convención metodológica.

Además, en orden a que nuestro sistema teórico goce del carácter empírico convendrá en cumplir con tres exigencias, a saber: 1) tiene que ser sintético, de tal forma que pueda constituir un mundo posible;19 2) debe lograr respetar el criterio de demarcación, lo que implica que no puede ser metafísico, sino que debe expresar un mundo lógicamente posible el cual es, también, empíricamente posible. Esto implica que la sinteticidad del sistema teórico debe de ser no metafísica. ${ }^{20} 3$ ) Finalmente está obligado a ser un sistema que pueda distinguirse de otros similares, en tanto que sería el sistema que ejemplifique nuestro mundo de experiencia (Popper, 2004, pp. 38-39). ${ }^{21}$ Dicha distinción podremos realizarla gracias a la contrastación a la que ha sido sometido el sistema y su supervivencia a la misma.

La disciplina histórica con la que lidiamos no está exenta de lo que entendemos como mundo posible. Lidiamos con documentos y obras elaboradas dentro del mundo de experiencia posible, por lo tanto no deberíamos desviarnos de los objetos que pueden posibilitar la corroboración de hipótesis y la formulación de hipótesis falsadoras. Nuestras hipótesis que se encuentren inmersas en la historia del arte,

cos conceptuales fueron desarrollados y sustituidos por otros nuevos y mejores; somos nosotros quienes creamos nuestras 'prisiones' y quienes también mediante la crítica, las destruimos" (Lakatos, 1983, p. 32). El filósofo excluye de los activistas revolucionarios a Hegel, ya que para él y para quienes lo siguieron: “... el cambio de los marcos conceptuales es un proceso predeterminado e inevitable donde la creatividad individual o la crítica racional no desempeña una función esencial. En esta 'dialéctica' los que se adelantan son tan culpables como los que se rezagan. No es sabio el hombre que construye una 'prisión' mejor o el que críticamente destruye la antigua, sino el que siempre se acompasa con la historia. De este modo la dialéctica explica el cambio sin crítica" (Lakatos, 1983, p. 32). 18 Esto se contrapone a las teorías del conocimiento 'pasivas', las cuales “... mantienen que el conocimiento auténtico es la impresión de la naturaleza en una mente completamente inerte; la actividad mental sólo puede resultar en prejuicios y distorsiones" (Lakatos, 1983, p. 32).

19 No contradictorio (Popper, 2005, p. 38)

20 Debemos de tener claro que la base empírica de la ciencia no es una base indiscutible ni 'absoluta' (Popper, 2004, p. 106): “... la ciencia no está cimentada sobre roca: por el contrario, podríamos decir que la atrevida estructura de sus teorías se eleva sobre un terreno pantanoso, es como un edifico levantado sobre pilotes. Estos se introducen desde arriba en la ciénaga, pero en modo alguno hasta alcanzar ningún basamento natural o 'dado'. Cuando interrumpimos nuestros intentos de introducirlos hasta un estrato más profundo, ello no se debe a que hayamos topado con terreno firme: paramos simplemente porque nos basta que tengan firmeza suficiente para soportar la estructura, al menos por el momento" (Popper, 2004, p. 106).

21 Al final, las teorías científicas son nuestras herramientas para poder entender 'el mundo', “... para racionalizarlo, explicarlo y dominarlo" (Popper, 2004, p. 57). 
deben poder ser distintas y claras, cada una sintética, las cuales faciliten una aproximación al mundo de experiencia posible. Ahora bien, debe quedar claro que tienen que ser sintéticas no metafísicas, o sea, sintéticas a posteriori, y no a priori; su carácter a posteriori posibilita que su validación suponga al menos una experiencia humanamente posible.

Con lo dicho hasta el momento, no queremos que haya confusiones respecto de si nuestra propuesta es un intento de suplantar las metodologías y teorías existentes en la historia del arte. La pretensión de establecer la disciplina como una ciencia empírica se da con el fin de poder determinar, posteriormente, cuáles metodologías están incluidas en el juego del conocimiento. Para ello dichos sistemas tienen que estar en condiciones de formular cualquier discusión o conjunto de argumentos que sean de talante racional. En este sentido Popper (2004, p. 17) establece un postulado metodológico que debe ser empleado por toda ciencia si quiere ganar el perfil racional, "(...) me refiero al de enunciar claramente los propios problemas y de examinar críticamente las diversas soluciones propuestas." (Popper, 2004: 17). En este caso los conceptos 'racional' y 'crítico' son equivalentes, ya que las soluciones planteadas serán atacadas, de forma crítica, por su creador. Cuanta mayor claridad haya en el enunciado de la pregunta, y en la definición de la solución, con más facilidad se podrá discutir críticamente al respecto. Al final no importa, y no nos importa en este texto, qué método relativo a la historia del arte utilice el investigador, siempre que haya un problema sin resolver, y exista honestidad intelectual a la hora de solventarlo.

\section{La historia del arte al amparo de los programas de investigación científica}

En conformidad con Lakatos (1983, p. 13), el criterio de demarcación de Popper, tanto para delimitar las teorías empíricas y las metafísicas como para separar de forma clara y distinta la ciencia y la pseudo ciencia, no es del todo satisfactorio, por lo que propone su teoría de la metodología de los programas de investigación científica. Esta no solo permite resolver ciertos conflictos planteados por Popper, sino también por la alternativa al filósofo austro-británico, liderada por el filósofo de la ciencia Thomas S. Kuhn (1922-1966).22 Para el filósofo húngaro, en

\footnotetext{
22 Según el filósofo húngaro, Thomas Kuhn proponía ver las revoluciones científicas como un cambio de carácter irracional en cuanto a las convicciones del momento, una especie de conversión religiosa: "Para Kuhn, el cambio científico de un paradigma a otro es una conversión mística que no está ni puede estar gobernada por reglas racionales y que cae enteramente en el terreno de la psicología (social) de la investigación." (Lakatos, 1983, p. 19). Kuhn propuso esto en contra del falsacionismo de Popper, pero si le diéramos la razón a Kuhn, estaríamos afirmando que no hay tal cosa como una demarcación entre ciencia y pseudo ciencia, y que no estamos en capacidad de distinguir el progreso científico de la decadencia intelectual (Lakatos, 1983, p. 13). Para Lakatos (1983, p. 120), en la pro-
} 
vez de hablar de hipótesis o teorías aisladas, necesitamos referirnos a programas de investigación, conformados por un conjunto de teorías, y concentrarnos en si un programa de investigación es progresivo o regresivo. En el primer caso, el programa nos permite conocer hechos nuevos que hasta el momento eran desconocidos, en el segundo el conjunto de teorías solo funcionan para ordenar o reordenar los hechos que ya conocemos. Lakatos nos pide tratar con benevolencia los programas de investigación en desarrollo, los cuales ya de por sí, como él mismo reconoce, nacen falsados, y que puede pasar mucho tiempo antes que estos se conviertan en programas progresivos de carácter empírico (Lakatos, 1983, pp. 15-16). Si la teoría está inmersa en un programa de investigación, el daño más remoto que podría recibir es que se convierta en un programa regresivo, estado del cual puede salir si logra producir nuevos hechos.

Nada de esto significa que Lakatos elimine el criterio de falsabilidad y por lo tanto el falsacionismo metodológico de Popper, sino que los modifica y lo inserta en sus programas de investigación bajo el título de falsacionismo metodológico sofisticado. Consideraremos por lo tanto una teoría como científica (o aceptable) solamente en el caso de que esta posea un exceso de contenido empírico en parte corroborado con relación a su antecesora, o contrincante. Esto completaría nuestro criterio de demarcación para la comprensión de la historia del arte como ciencia. La teoría debe permitirnos descubrir nuevos hechos (Lakatos, 1983, pp. 46-47):23

Para el falsacionista sofisticado una teoría científica T queda falsada si y sólo si otra teoría T' ha sido propuesta y tiene las siguientes características: 1) T' tiene un exceso de contenido empírico con relación a $\mathrm{T}$; esto es, predice hechos nuevos, improbables o

puesta de Kuhn: “... las anomalías y las inconsistencias siempre abundan en la ciencia, pero en los períodos 'normales' el paradigma dominante asegura una pauta de crecimiento que eventualmente es destruida por una 'crisis'. No existe una causa racional particular para la aparición de una 'crisis' kuhniana. 'Crisis' es un concepto psicológico; se trata de un pánico contagioso. Después aparece un nuevo paradigma que es inconmensurable con relación a su predecesor. No existen criterios racionales para compararlos. Cada paradigma contiene sus propios criterios. La crisis arrastra tras de sí no sólo las viejas teorías sino también los criterios que hacían que los respetáramos. El nuevo paradigma trae consigo una racionalidad completamente nueva. No hay criterios superparadigmáticos. El cambio tiene efectos acumulativos. Por tanto, y según Kuhn, las revoluciones científicas son irracionales, objeto de estudio de la psicología de masas.

Lakatos (1983, p. 15) explica de esta forma las revoluciones científicas: "Si tenemos dos programas de investigación rivales y uno de ellos progresa, mientras que el otro degenera, los científicos tienden a alinearse con el programa progresivo. Tal es la explicación de las revoluciones científicas." Posteriormente, el filósofo aclara que no es un crimen por parte del científico aferrarse a un programa regresivo, ya que este, en cuanto programa, está en posibilidad de convertirse en progresivo, y esto gracias al investigador" (Lakatos, 1983, p. 16).

${ }_{23}$ Contrario al falsacionista metodológico ingenuo, en donde sólo se necesita que las teorías sean consideras como falsables, es decir, contrastables, para que las categoricemos como científicas (Lakatos, 1983, pp. 46-47). 
incluso excluidos por T; 2) T' explica el éxito previo de T; esto es, todo el contenido no refutado de $\mathrm{T}$ está incluido (dentro de los límites del error observacional) en el contenido de T', y 3) una parte del exceso de contenido T' resulta corroborado. (Lakatos, 1983, pp. 46-47)

El carácter progresivo de un programa de investigación lo podemos establecer en dos partes, una teórica y la otra empírica. En el caso de la primera, el programa de investigación será teóricamente progresivo si nos lleva a nuevas predicciones, y será empíricamente progresivo si algunas de dichas predicciones llegan a ser corroboradas (Lakatos, 1983, p. 230).

Por último, con relación a la propuesta lakatosiana que queremos adoptar, los programas de investigación se componen de reglas metodológicas, estas se dividen en la heurística negativa, en donde están contenidas las rutas de investigación que han de ser evitadas, y la heurística positiva, que nos dice las vías que debemos seguir. Asimismo, el programa de investigación tiene dos partes, el centro firme y el cinturón protector. La heurística negativa no permite que sometamos a contrastación al centro firme, en él están contenidas las rutas que han de ser evitadas (Lakatos, 1983, pp. 65-66). El centro firme de un programa se vuelve, por lo tanto, 'irrefutable' por decisión metodológica (acuerdo) entre sus defensores (Lakatos, 1983, pp. 68-69). Entonces, es nuestra misión establecer un cinturón protector, esbozado por la heurística positiva, que resguarde el centro firme, edificado por hipótesis auxiliares, quienes serán objeto de contrastación (falsación metodológica) dentro del programa de investigación (Lakatos, 1983, pp. 65-66). El cinturón protector es la parte refutable, o mejor dicho, contrastable del programa de investigación. La heurística positiva al final lo que nos da son las posibilidades para comprometer al cinturón protector el cual, en orden a ser progresivo, tiene que realizar, ante la contrastación, una serie de ajustes que produzcan nuevos hechos (Lakatos, 1983, pp. 68-69):

El cinturón protector de hipótesis auxiliares debe recibir los impactos de las contrastaciones y para defender al centro firme, será ajustado y reajustado e incluso completamente sustituido. Un programa de investigación tiene éxito si ello conduce a un cambio progresivo de problemática; fracasa, si conduce a un cambio regresivo. (Lakatos, 1983, p. 66)

La forma en que el centro firme puede resquebrajarse no es por medio de un ataque directo, sino en el momento en que el programa deja de proporcionar hechos nuevos (Lakatos, 1983, p. 68). Esta producción depende del cinturón protector y los procesos de falsación, por lo tanto sus ajustes deben de ser consistentemente progresivos en función de evitar caer en un reacomodo constante que no produzca nada nuevo. Ahora bien, un programa de investigación regresivo puede convertirse en 
uno progresivo, la decisión de abandonar (no eliminar) un centro firme depende del estado del cinturón protector, y de las acciones a tomar al respecto por parte del investigador. Dicho abandono requiere la presencia, a su vez, de un nuevo programa de investigación, que, como ya hemos aludido anteriormente, al competir con el rival, haya podido explicarlo y producir un exceso de contenido. Cabe recalcar que nadie está en obligación de afiliarse a un programa de investigación hasta el final, es decir, hasta que este se haya estancado o agotado en cuanto a su heurística positiva. Tampoco debemos esperar a que un programa se vuelva regresivo para crear un programa de investigación rival (Lakatos, 1983, p. 92).

A partir de Lakatos y su concepción del falsacionismo metodológico popperiano, podemos ampliar nuestra definición de historia del arte, y verla, en calidad de ciencia, como un conjunto de programas de investigación en competencia que lidian con el objeto artístico como problema histórico. Al mismo tiempo, los programas se componen de una serie de teorías en las cuales, por vía de la determinación de sus heurísticas negativa y positiva, podemos distinguir el centro firme y el cinturón protector. Las teorías pueden proporcionar ya sea un cambio progresivo o uno regresivo de problemática. Es necesario puntualizar que los programas de investigación deben encontrarse en competencia, ${ }^{24}$ ya que nuestra finalidad es poder progresar en cuanto al conocimiento, circunscrito en la noción de mundo de experiencia posible.

Si visualizamos la historia del arte como programas de investigación, podemos reconstruir, de forma racional, ciertos procesos que han ocurrido a través de la historiografía de la disciplina, en la que diversos programas se encuentran en competencia, y cada uno por su lado, ha ofrecido cambios progresivos y regresivos de problemática. A continuación mostraremos un breve ejemplo de la posibilidad del uso de los programas de investigación científica dentro de la historia del arte, para aproximarnos, de forma racional, a diversos procesos que han ocurrido a lo largo de la disciplina.

\section{La Madona del burgomaestre Meyer: el camino de una ciencia según Kultermann}

Nos referiremos al trabajo que realizó el historiador del arte Udo Kultermann relativo a la Madona del Burgomaestre Meyer de Hans Holbein el Joven (c. 14971543), en su libro Historia de la historia del arte: el camino de una ciencia. Kultermann (1996, pp. 193-194) nos habla de una discusión que se llevó a cabo a partir del Congreso de Dresde sobre Holbein, en septiembre de 1871, en torno al

${ }^{24}$ La historia es, ante todo, “... una historia de programas de investigación que compiten (...) cuanto antes comience la competencia tanto mejor para el progreso" (Lakatos, 1983, p. 92). 
método de examen artístico, metodología la cual utilizaba disímiles fuentes literarias para analizar y autenticar las obras. ${ }^{25}$ La polémica se suscitaba a partir de la atribución de 46 pinturas a Holbein, de las cuales, por vía de una historia del arte diferenciada del método del examen artístico, se rechazó la autoría del artista en aproximadamente 32 de ellas. En esta serie de trabajos se tomaba en cuenta a la Madona del Burgomaestre de Meyer de la que había dos ejemplares, uno en Dresde, que era el que pertenecía a las 46 pinturas, y otro en Darmstadt. Los historiadores del arte lograron concluir que la obra original era la de Darmstadt, realizada en 1526, y que la de Dresde era una copia elaborada en el siglo XVII por Bartholomäus Sarburgh.

La determinación del trabajo de Dresde como una copia, en conjunto con el rechazo de la mayoría de obras generó una serie de polémicas que ponían en crisis los métodos de atribución. ${ }^{26}$ Kultermann se dispuso a explicar cómo el nuevo método propulsado por la historia del arte se impuso victoriosamente sobre el método de examen artístico dentro de la historiografía del arte, proceso que podemos traducir a dos programas de investigación en competencia en el que uno se convirtió en progresivo, y el otro en regresivo.

Quienes estaban en contra de lo expuesto por los historiadores del arte se amparaban ante una supuesta belleza superior que tenía el cuadro de Dresde, y cómo era un trabajo que superaba en este sentido al de Darmstadt, entonces debía atribuirse a Holbein, elevando al artista como el gran maestro de uno de los objetos más importantes del arte alemán. Considerar el trabajo de Darmstadt como el original era rebajar la maestría del artista alemán a un arte de menor calidad. Dentro de este grupo nos encontramos al filósofo, físico y psicólogo Gustav Theodor Fechner (1801-1887), quien publicó en 1871 su trabajo Über die Echtheitsfrage der Holbein'schen Madonna, ${ }^{27}$ en el que aseguraba que el cuadro original era el de Dresde, y que en todo caso este era anterior al de Darmstadt. Dentro de la tradición del método de examen artístico elaborado solamente a partir de la observación y un gran conocimiento en literatura y en arte, Fechner establecía que la autenticidad el

\footnotetext{
25 En conformidad con Kultermann (1996, pp. 193-194): "En los primeros tiempos de la Historia del Arte, las atribuciones eran, por regla general, erróneas. Por ejemplo, la Venus durmiendo de Giorgione de Dresde fue considerada durante mucho tiempo como una obra de la escuela del maestro y, de vez en cuando, también como una copia en el estilo de Tiziano hecha por Sassoferrato. Giovanni Morelli fue quien demostró que el cuadro era una de las obras capitales de Giorgione. El Retrato de Morett de Holbein, igualmente en la Galería de Dresde, se consideró durante bastante tiempo como un Leonardo da Vinci. Rumohr fue el primero que sacó la obra del ámbito de Leonardo y la atribuyó a Holbein." 26 Y no estaba de más la polémica, ya que “... la copia de Sarburgh (...) fue festejada como reliquia nacional, comparándosela con la Madonna Sixtina de Rafael como obra de la misma categoría" (Kultermann, 1996, p. 196).

27 Sobre la cuestión de la autenticidad de la Madonna de Holbein, traducción de Kultermann (1996, p. 197).
} 
cuadro solo se podía determinar a partir de una cierta consideración romántica particular de belleza (Kultermann, p. 1996, pp. 197-198);28 es decir, a lo interno del programa de la atribución se establecía como centro firme la posibilidad de autenticar obras a través de nociones sentimentales literarias ajenas a las artes plásticas, que se vinculaban de alguna forma a la maestría del pintor. La manera en que se protegía este argumento era con un corpus de obras que tenían ciertas afinidades estéticas justificadas por medio de discursos románticos literarios utilizados de forma deliberada, y que con ellos los trabajos podían ser clasificados por sus similitudes dentro del trabajo de un artista.

La evaluación que hicieron los historiadores del arte demostró un progreso respecto de esta perspectiva, adoptada en un principio por la misma historiografía del arte, ya que se creaba un método que se concentraba en la obra individual, y se olvidaba de una libre interpretación literaria que pretendía acomodar todos los elementos a su favor. Ya desde 1844 el historiador del arte Franz Kugler (1808-1858) proponía ciertas dudas respecto a la atribución que se hacía del cuadro de Dresde, al notar una estilización en la cabeza de la Virgen, y una serie de tonos verduscos en la piel, que no encontraba en los trabajos conocidos del pintor alemán. El historiador del arte Gustav Friedrich Waagen (1794-1868) apoyaba lo expuesto por Kugler, aunque no eliminaba la posibilidad de que la Madona de Dresde fuese original, y veía en la pintura de Darmstadt un tratamiento y ejecución más característicos de Holbein. A estas nociones se unió Albert von Zahn (1836-1873), también historiador del arte, quien estuvo involucrado en el conflicto de 1871, y que concluía, tras un análisis detallado, que sin duda la Madona de Darmstadt era original. Además, sale a relucir el libro de Nicholas Ralph Wornum, Some Account of the Life and Works of Hans Holbein, ${ }^{29}$ publicado en 1867, quien declara la pintura de Dresde como una copia de menor calidad. Finalmente contamos con lo manifestado por los historiadores Bruno Meyer (1840-¿?) y Karl Woermann (1844-1933), quienes, al igual que Zahn, participaron activamente en el evento del 71 (Kultermann, 1996, pp. 198-199, 201). ${ }^{30}$ Un año antes Meyer exponía:

\footnotetext{
28 Tal era la explicación del escritor Herman Grimm (1828-1901) (en Kultermann, 1996, p. 200): "No me explico cómo se puede atribuir la pintura de Dresde a cualquier otro que no sea Holbein. El otoño pasado estuve en Darmstadt en tres ocasiones y durante varias horas ante la obra. En la mano tenía una fotografía de la Virgen de Dresde para comparar línea a línea. Y línea a línea se podía reconocer, tanto en el dibujo como en la disposición de los grupos, un avance hacia cotas más sublimes, digámoslo así, más ideales, que sólo pudo hacer el propio maestro."

29 Algunas observaciones sobre la vida y las obras de Hans Holbein, traducción de Kultermann (1996, p. 199).

30 Entre el grupo de expertos de dicho evento nos encontramos a Alfred Woltmann (1841-1880), Moritz Thausing (1838-1884), Karl von Lützow (1832-1897), Adolf Bayersdorfer (1842-1901), Friedrich Lippmann (1838-1903), Wilhellm Lübke (1826-1893), Bruno Meyer, Karl Woermann, G. Malz (s.f.) y Wilhelm von Bode (1845-1929). Ellos fueron quienes escribieron el comunicado oficial
} 
$\mathrm{Si}$, como es de esperar, la comparación de ambas Vírgenes, proyectada para este otoño, se lleva a cabo en Dresde en el año en curso, de aquí en adelante la Virgen "de Holbein" de Dresde sólo pervivirá en la Historia del Arte como el interesante monumento de un largo error. (Meyer en Kultermann, 1996, p. 201)

A su vez, en 1873 Woermann se refirió a los sucesos de esta manera:

La famosa Virgen del burgomaestre Meyer (1525) de Darmstadt (...) como cuyo original fue elogiada la bella y algo modificada copia de Dresde antes de las aclaraciones de Zahn, Woltmann, Bayerdorfer y el autor de este libro, ha sido reconocida hace tiempo como el único cuadro de la mano de Holbein. La copia de Dresde, según demostró Ernst Major, probablemente fue ejecutada en torno a 1636 en Ámsterdam para la reina francesa María de Medici por su pintor de corte Bartholomäus Sarburgh.

En términos generales, podemos decir que los investigadores argumentaban que se debía hacer un análisis de la obra en concreto, ignorando la intuición contemporánea vinculada al espíritu, lo majestuoso, y la literatura ajena a la misma obra. Este análisis se realizaba a partir de una historia del estilo tanto de la época de Holbein, como una historia del estilo de la obra de Holbein. Los historiadores consideraban que era posible determinar la autenticidad de un objeto artístico a partir de un conocimiento técnico-histórico con el que fuese posible someter a contrastación la obra, y así se mostrasen similitudes o diferencias técnicas y estilísticas (centro firme). Esta hipótesis era protegida con investigaciones relativas a cuadros o esculturas en particular en las que se demostraba la efectividad de tal método. Su éxito residía en que contaba con fuentes primarias con las cuales corroborar o falsar la atribución, a diferencia del examen artístico, cuyo conocimiento se reducía a exigencias particulares que la época tenía respecto de la estética y el espíritu del arte, en este caso el arte alemán. Con esto tomado en cuenta, era más sencillo comparar las dos obras, y concluir que la que se aproximaba más al trabajo de Holbeinera la de Darmstadt.

De esta manera, el programa de investigación que articulaban los historiadores ya por varios años es progresivo, en tanto que logró generar un exceso de conocimiento antes no existente respecto de las obras de arte como problemas históricos. El conocimiento producido hacía del programa en competencia, es decir, del método del examen artístico, un programa regresivo, en el que se acomodaban diversos datos y estos se sometían a exigencias estéticas de una época en particular. Este trabajo no ofrecía nada nuevo respecto de la obra de Holbein, más bien estaba haciendo atribuciones que hoy podemos considerar falsadas, y que en todo caso perjudicaban nuestro conocimiento relativo al artista alemán.

en 1871, que establecía como obra original de Holbein el cuadro de Darmstadt, y no el de Dresde (Kultermann, 1996, pp.196-197). 


\section{Conclusión: una nueva definición de historia del arte como ciencia}

A manera de conclusión, y a la luz de lo desarrollado en este artículo, presentemos una vez más nuestra proposición de definición de historia del arte. Entenderemos a la historia del arte, en tanto ciencia, como un conjunto de programas de investigación que se encuentran en constante competencia por la generación de cambios progresivos de problemática, que proporcionan como resultado colateral cambios regresivos en los programas rivales. Estos programas de investigación son, al mismo tiempo, un complejo de teorías relativas al estudio de los objetos artísticos como problemas históricos, y, por medio de la determinación de la heurística negativa y la heurística positiva del programa, podremos especificar el centro firme, irrefutable por convención, y el cinturón protector, el cual a través del falsacionismo metodológico sofisticado, se convierte en el campo para la producción de conocimiento, y le posibilita al programa ganar tanto el carácter teóricamente progresivo, como el empíricamente progresivo.

Lakatos (1983, p. 173) nos ofrece la posibilidad de realizar una reconstrucción racional de la ciencia, y nos alerta en que no debemos aspirar de forma inmediata a la construcción racional de la historia, ya que: "Las reconstrucciones racionales siempre estarán sumergidas en un océano de anomalías. Tales anomalías habrán de ser explicadas eventualmente bien por alguna reconstrucción racional mejor o por alguna teoría empírica 'externa'." (Lakatos, 1983, p. 173).

Nos gustaría, de esta manera, terminar nuestro ensayo con una última cita de Lakatos:

(...) el progreso en la teoría de la racionalidad científica está jalonado por descubrimientos de nuevos hechos históricos, por la reconstrucción de un cúmulo creciente de historia impregnada de juicios de valor, como racional. En otras palabras, la teoría de la racionalidad progresa si constituye un programa de investigación historiográfico 'progresivo' (Lakatos, 1983, p. 173).

\section{Referencias bibliografícas}

Agassi, J., y Jarvie, I. (2008): A Critical Rationalist Aesthetics, Amsterdam-New York, Rodopi.

Kultermann, U. (1996): Historia de la Historia del Arte: El Camino de una Ciencia, Madrid, Akal Ediciones.

LAKATOS, I. (1983): La metodología de los programas de investigación científica, Madrid, Alianza Editorial, S. A.

Oviedo, M. (2014): Per Monstra ad Sphaeram: La función del arte de la estampa en la concepción de Aby Warburg del proceso de liberación astrológica en épo- 
cas del Renacimiento y la Reforma (Tesis de Licenciatura). Universidad de Costa Rica, San José.

PANOFSKY, E. (2008): "On the Relationship of Art History and Art Theory: Towards the Possibility of a Fundamental System of Concepts for a Science of Art (1925)", Critical Inquiry, 35, pp. 43-71.

PopPER, K. R. (2004): La lógica de la investigación científica, Madrid, Editorial Tecnos.

PopPeR, K. R. (2006): La miseria del historicismo, Madrid, Alianza Editorial.

Mauricio Oviedo Salazar

Universidad de Costa Rica

mauricio.oviedo.salazar@gmail.com 